\title{
Fractional weighted ZF equalizer: A novel approach for channel equalization in MIMO-OFDM system under impulse noise environment
}

\author{
S.P. Girija ${ }^{a, *}$, Rameshwar Rao ${ }^{\text {b }}$
}

\author{
${ }^{a}$ Department of Electronics and Communication Engineering, Kakatiya Institute of Technology and Science, Telangana 506009, India. \\ ${ }^{b}$ Department of Electronics and Communication Engineering, University College of Engineering, Osmania University, Telangana 506009, India.
}

Article history:

Received: 26 October 2020 / Received in revised form: 24 April 2021 / Accepted: 5 May 2021

\begin{abstract}
Impulse noise is the major factor degrading the performance of the wireless system, imposing the need for the impulse noise mitigation strategy. Mainly, the multiple-input multiple output (MIMO) and orthogonal frequency-division multiplexing (OFDM) system contaminated with the impulse noise creates major impact on the performance as the conventional zero-forcing (ZF) equalizer does not give satisfactory results. Thus, this paper concentrates on the impulse noise mitigation strategy based on the fractional weighted zero-forcing (FWZF) equalizer, which is the integration of the fractional concept in the Zero-Forcing equalizer. The noise impacts on the MIMO-OFDM system were minimized and the performance was enhanced due to the usage of the fractional theory in the ZF equalizer as the equalization values of the previous instances were interpreted for the formulation of the effective equalization value in the current instance of the ZF equalizer. The performance of the methods was done based on the valuation metrics, Bit Error Rate (BER) and Symbol Error Rate (SER) related to the Signal-to-Noise Ratio (SNR) and dissimilar antenna array size. It has been found that the proposed Fractional Weighed Zero-Forcing equalizer outperformed the existing methods with a minimal BER and SER of 0.063, and 0.1038, while analyzing the methods in the Rayleigh environment.
\end{abstract}

Keywords: MIMO-OFDM; impulse noise; equalizer; channel equalization; fractional concept

\section{Introduction}

With the extensive growth in the mobile data transmission over the wireless communication channels with higher transmission rates, there are various transmission techniques for MIMO system, which marks the significance of communities in the field of signal processing as well as communications [1]. For transmitting the signals over the wireless channels, the MIMO system with the OFDM turns out as the effective method. The implementation of OFDM is found in a number of wireless standards, like digital audio broadcast and some other approaches. Considering the prevailing issues in the channels, OFDM along with MIMO is found as the effective standard for communication over wireless in the next generation [2]. The potential of the system in enhancing the spectrum efficiency during communication is handled effectively using multiple array of antennas both at the transmitting and receiving terminals that assures the tendency to meet the higher data rates in the wireless communications. More particularly, MIMO systems attain greater interest in the current few years [3]. OFDM is a peculiar technique employed in the wideband wireless communication as a result of their higher data rates, spectral efficiency, and good quality of data transmission and the approach finds valuable application in numerous communication standards, like digital audio broadcasting (DAB), broadband wireless access standard,

\footnotetext{
* Corresponding author.

Email: spgirija@gmail.com

https://doi.org/10.21924/cst.6.1.2021.224
}

broadband wireless local area networks (WLANs) in addition to digital video broadcasting (DVB) [4]. MIMO communication, therefore, provides greater capacity and improves the performance in the extensive growing modern wireless telecommunication systems, like WiMAX, LTE, and Wi-Fi. The exponential enhancement in the performance corresponding to the MIMO systems, however, reports a highly expensive and complex signal processing at the receiver. Thus, the importance of the effective usage of MIMO relies on the presence of highly integrated and affordable terminals. The aforementioned factors raise the need for the establishment of the efficient receivers to enable an effective communication in future trending communication systems [5].

The enactment of digital communication systems engaged in WANs is reduced by adverse channel conditions and interferences, modeled as non-Gaussian impulsive noise. Even though multicarrier modulations engaged in the wireless communication systems are more opposed to impulsive noise, the performance degradation caused by impulse noise still becomes a challenging research area for communication engineers. There are number of impulse noise suppression methods introduced in the past [6].Yang Chen, et al. [7] introduced an iterative Structure-Adaptive Fuzzy Estimation (SAFE) aimed at random-valued impulse noise suppression. The SAFE approach was developed in the Gaussian Maximum Likelihood Estimation (GMLE) framework. The advantage of this approach is that it had good performance in both structure restoration and noise suppression for high noise densities. However, it required more acceleration for real-time computing. Hongqing Liu, et al. [8] explored signal sparsity in 
the frequency domain to estimate frequency under impulsive noise. This estimate of the sparse frequency was an effective technique for suppressing the impulsive noise. The click of the button purely was not an impulse and collected some samples based on the sampling frequency and duration of the pressed button. This feature has not been explored in this approach. Hossein, et al. [9] developed a technique to suppress the noise of high density impulses in real time from images. This technique surpassed the best existing techniques in terms of visual quality and PSNR measurement and was quite applicable for real-time applications. Bansal and Kohli [10] introduced the orthogonal frequency division multiplexing system (OFDM), operated with the Nakagami-m multipath fading channels and using the impulsive noise excision method. In this method, BER performance was degraded when a channel estimator error increased. Nikola Rožić, et al. [6] developed a nonlinear estimator dependent upon the multiple threshold with attenuation of the associated segment, or clipping of the amplitude of the received signal. These mitigation and clipping estimators unsurprisingly performed similarly to the Optimal Bayesian Estimator (OBE).

As bandwidth increases, multipath distortion or frequency selective fading of the physical medium continues to be weaker. The multipath channel leads to the time dispersion in the transmitted signal, causing a strong impact on the end of the receiver, which is reported as the superposition of numerous transmitted symbols. The aforementioned overlap is often called as Inter-Symbol Interference (ISI). A solution to solve the ISI problem is related to the receiver modeling. The receiver consists of compensating equipment to minimize the ISI present in the received signal and this compensation is initiated before detection and the compensator is referred as the equalizer. One of the equalizers is linear equalizer, which is widely employed in many applications due to its simple structure and minimal complexity. Additionally, the presence of noise is increased when suppressing the ISI. It is important to note that the impulsive noise is the major source of degrading the performance in the wireless systems. The traditional linear $\mathrm{ZF}$ equalizer is ineffective as the variation of the diversity order is not found to rise monotonically regarding the signal-to-noise ratio (SNR) [2]. However, in addition to the multipath fading, non-Gaussian or impulsive noise formed from the natural environments, such as atmospheric noise and artificial sources, such as ignition noise remains the dominant reason for degrading the performance in numerous wireless applications, in addition to the sensor networks. The deep insight over the existing literature reports that the design of the underlying channels is made based on the frequency-flat Rayleigh blockfading systems, and the noise samples of Middleton Class-AAmplifier (MCA) on the receiver side is regarded as either statistically dependent or independent, but they are spatially uncorrelated. Additionally, the computational capability is limited indicating that the application of the equalization structures affords low-complexity [11]. For the joint counteraction of the effects associated with an MCA additive noise and frequency-selective fading, an effective answer is adopted at the receiver part of a simple linear equalizer. When the impulse noise is present, the performance of the conventional zero forcing ( $\mathrm{ZF}$ ) algorithms is poor due to no particular measure to counteract the effects of noise. The basic shortcoming associated with the linear equalizer while coping with the severe ISI stands as a motivation behind developing enormous research regarding the suboptimal nonlinear equalizers, which possesses minimal computational complexity, like decision feedback equalizer (DFE) [12,13]. The highly available challenge regarding the MIMO receiver is related to the complexity and the main role of the MIMO channel equalizer / detector is related to the separation of spatially multiple data flows on the receiver terminal. Channel equalization in MIMO is performed through a variety of methods. Of the available methods, Vertical Bell Labs layered space-time (V-BLAST) architecture [16] is used as an effective method. It deals with narrow band MIMO channels and effectively eliminates interference between (sub) channels, thus improving the reliability of symbol detection. To improve the robustness and throughput of communications in spacetime frequency selective channels, transmission in MIMO is performed in combination with turbo equalization (TEQ), which is the iterative equalization and decoding method recognized as the effective solution in UWA communications [14].

There are numerous methods developed for signal separation, such as equalization of MIMO decision feedback equalization [15], Bell Labs layered space-time (BLAST) architecture [16], blind MIMO equalization [17], or Adaptive MIMO equalization [18]. Most of the assumptions concern the quasi static nature of the channel, which is invariant over time in a data frame. On the other hand, the practical wireless channels are essentially variable over time, which varies within a data frame generating CCI on the received signals and produces a low bit error rate (BER), which degrades performance at receiving terminal [3]. For establishing channel equalization in the system, Massive MIMO was used in [19]. To address the problems mentioned above, adaptive Frequency Domain Equalization (FDE) methods were used depending on the Recursive least squares (RLS algorithm and Least Mean squares (LMS) algorithms in MIMO systems [20,21]. On the other hand, it has been found that the performances corresponding to the adaptive algorithms are not satisfactory in the case of fast fading channels which vary over time due to the weak correlation in the channel at various times, mainly in the doubly selective channels. . The Iterative Block Decision Feedback Equalizer (IB-DFE) enables an excellent performance to approach the combined filter limit (MFB) in addition to MIMO systems. On the contrary, in the case of MIMO-SCFDE systems, they used doubly selective channels; the joint estimation of the channel and equalization algorithms (JCEE) depended on the IB-DFE concept, which is not addressed in the literature [22].

The major contribution of the research: Proposed Fractional weighted Zero Forcing Equalizer: The fractional weighted Zero-Forcing (ZF) equalizer was developed through integrating the prediction model based on the fractional weight in the existing $\mathrm{ZF}$ equalizer that compensated for the channel effect on the MIMO-OFDM systems.

The rest of the paper is structured as: The proposed method of noise cancellation is detailed in Section 2 under materials and methods; Section 3 discusses the existing methods along with the merits and demerits and demonstrates the results of the methods. Finally, Section 4 concludes the paper.

\section{Materials and Methods}

The present research concentrated on the impulse noise, a significant factor for performance degradation in the MIMOOFDM. The investigation modelled a technique for equalizing the channel in MIMO-OFDM system using the FWZF equalizer in the impulse noise environment. The proposed FWZF equalizer is the modification of the linear ZF equalizer with the integration of the fractional weight in such a way that 

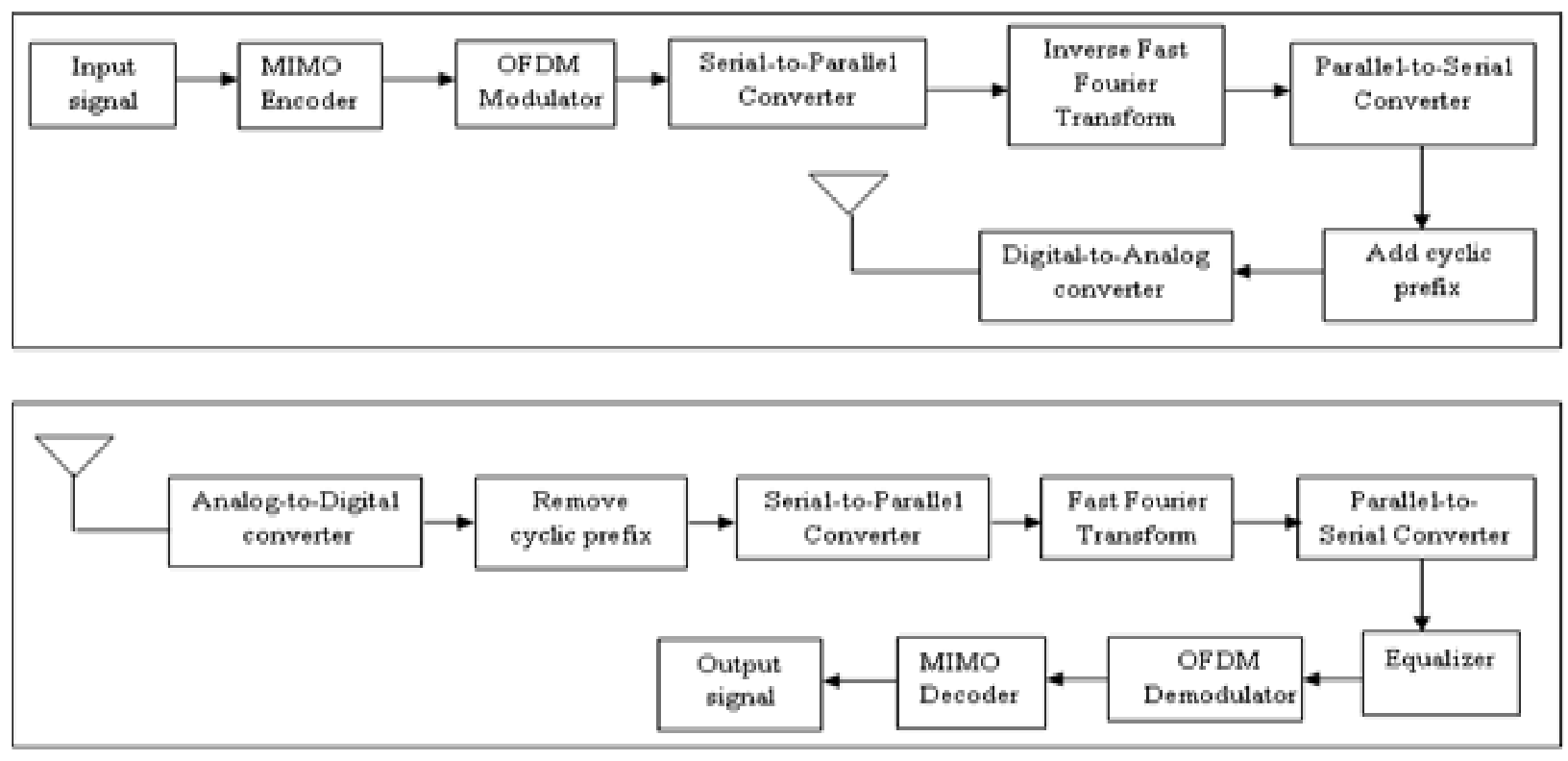

Fig. 1.Depicts the MIMO-OFDM block diagram

the prediction model based on the fractional theory integrated the equalization values of the previous instances in determining the equalization value of the current instance. Thus, the zero-forcing blind equalizers with fractional weightbased delay were used directly to estimate the second-order statistics of the records. Overall, the input signal was directly sent to the transmit filter to remove the impulse noise, which was then forwarded to the fading channel. Upon receiving the signal in the receiver, the filter in the receiver compensated the impulse noise through the application of the fractional weighted $\mathrm{ZF}$ equalizer that compensated for the channel effect.

\subsection{MIMO-OFDM system model}

The MIMO-OFDM system was made up of several transmissions and reception antennas equipped respectively with $A_{T}$ transmission and $A_{R}$ reception antennas. It is peculiar to observe that the number of receiving antennas is not less than the total of transmitting antennas, indicated as $A_{R} \geq A_{T}$ The transmitter was allowed to demultiplex the input data followed by coding the data to generate symbol streams through serialparallel conversion. Then, the symbol streams were quickly entered into the constellation mapping, followed by modulation using the IFFT (Inverse Fast Fourier Transform) modulators and there was a cyclic prefix. Finally, $A_{T}$ OFDM signals were transmitted through the transmitter antenna. At the same time, at the end of the receiver, the individual antennas of the receiver received signals from all transmission antennas. Before receiving the signals on the receiver antenna, there were a few steps. Initially, the added cyclic prefix was removed, followed by parallelization. Then, FFT was applied and demodulated to allow signal reception on the receiver antenna. Figure 1 lists the schematic diagram of MIMO-OFDM. The antenna of the individual transmitter transmitted the data symbols in the form of data vectors,

$$
G^{h_{T}}=\left(G_{1}^{h_{T}}, G_{2}^{h_{T}}, \ldots, G_{m}^{h_{T}}\right)^{T}, h_{T}=1, \ldots, D_{T}
$$

where, $m$ symbolizes the total data symbols and each of the data vectors comprises of $m$ data symbols. The OFDM symbols were transmitted over the transmit antennas and the received signal was modeled for all sub-carriers. The receiver signal for the $h^{\text {th }}$ sub-carrier is modeled as,

$$
S_{d}^{h_{l}}=\sum_{h_{T}=1}^{D_{T}} C_{d, d}^{\left(h_{l}, h_{T}\right)} G_{d}^{D_{T}}+N_{d}^{h_{l}}
$$

where, $d$ specifies the diagonal element and there is $m$ number of diagonal elements. $C_{d, d}^{\left(h_{l}, h_{T}\right)}$ corresponds to the $d^{\text {th }}$ diagonal element in the diagonal channel matrix $C^{\left(h_{l}, h_{T}\right)}$ that links the transmitter antenna $h_{T}$ and the receiver antenna $h_{R}$. Equation (1) symbolizes the symbol vector transmitted that is of dimension $(g \times 1)$, where $g$ corresponds to the total transmit antennas. Similarly, $S(h)$ refers to the symbol vector acknowledged at the receiving antenna and the measurement of the symbol vector at the receiver side is denoted as $(f \times 1)$. The noise vector $N(h)$ specifies the impulse noise vector of dimension $(f \times 1)$ and the channel matrix for the channel of dimension $(f \times g)$ is represented as $C(h)$.

\subsection{Zero forcing $(Z F)$ equalizer}

It is linear equalization algorithm engaged in communication systems, functioning at the reversal of the frequency response corresponding to the channel. The foundation for the ZF equalizer was laid by Robert Lucky. At the receiver end of $\mathrm{ZF}$, the signal vector of the receiver $S$ was multiplied with the filter matrix. Let us denote the filter matrix as $F$ engaging in inverting the channel. Thus, the filter matrix is computed as

$$
F=C^{+}=\left(C^{+} C\right)^{-1} C^{C}
$$

where $C^{+}$refers to the generalized inverse matrix corresponding to the matrix $C$ and ()$^{C}$ refers to the Hermitian transpose function. For recovering the signal $S^{n}$, the received transmit signal vector $G^{n}$ was permitted to undergo the linear 
processing mechanism. The computation of the linear processing is given as,

$$
\hat{G}^{n}=H^{n}(C)^{n}
$$

Here equation (4) is the equalizer output of $G^{n}$. Finally, the entity output of the above outcome was quantized related to the adjacent symbols in $G$. The selection of the matrix $H^{n}$ is the ZF equalizer in MIMO-OFDM given by

$$
H_{Z F}=K^{\prime}
$$

It is significant to note that the $\mathrm{ZF}$ equalizer never operates perfectly in the presence of the impulse noise; hence, there is a need for the effective equalization strategy to operate effectively in the availability of the impulse noise and minimal mean output-energy (MMOE) condition. The method meeting the aforementioned key points renders the effective performance. As per condition $A_{R}>A_{T}$, the equalizer performs by minimizing the MMOE for $d^{\text {th }}$ symbol stream with respect to the $\mathrm{ZF}$ constraint. The MMOE is given as,

$$
\left.M M O E_{d}=\varepsilon\|G\|^{2} C\right]
$$

The $\mathrm{ZF}$ constraint is given as,

$$
\left.H_{Z F-M M O E}=\arg \min \varepsilon\|S\|^{2} C\right]
$$

Substitute the equation,

$$
H K=J_{A_{T}}
$$

The solution is represented as,

$$
\begin{gathered}
H_{Z F-M M O E}=\left(K^{C} \psi_{k k}^{-1} C\right)^{-1} K^{c} \psi_{k k}^{-1} \\
\psi_{k k}=\varepsilon\left[k k^{C}\right]=K K^{C}+\psi_{s s}^{A} \in K^{A_{R} \times A_{R}}
\end{gathered}
$$

Equation (10) represents the correlation matrix determined approximately using the received data. The effective model of the equalizer enabled the performance in the presence of the impulse noise and in this paper; the effective $\mathrm{ZF}$ equalizer based on the proposed Fractional weight was developed.

\subsection{Proposed fractional weighted equalizer for channel equalization}

The MIMO-OFDM system is modeled as indicated in the previous section. In this section; the fractional weighted $\mathrm{ZF}$ equalizer for the system is modeled and elaborated in detailed. The developed equalizer was able to reduce the impulse noise and at the equalizer output; the impact of noise was minimized. The wireless communication channel was designed as a linear model of the invariant impulse response over time. Depending on the overall architecture, there are many subcarriers and modulators on the individual subcarriers. In the communication system, the noise correlation matrix is considered essential to overcome the effects of noise on the system so that the effect of noise is effectively mitigated on the basis of this matrix. Therefore, in this paper, the fractional idea was integrated in the formulation of the noise correlation matrix which interpreted the values of the previous instances. At the output of the equalizer, the effect of noise was mitigated and the noise correlation matrix is modeled as follows:

$$
\psi_{s s}=\sigma_{s}^{2} J_{A_{R}}
$$

where, $\sigma_{s}^{2}$ represents the variance and $J_{A_{R}}$ specifies the identity matrix. To enhance the performance of SNR in the MIMO-OFDM system, the noise correlation matrix is rewritten as follows:

$$
\psi_{s S}=\frac{1}{2} \sigma_{S}^{2} J_{A_{R}}+\frac{1}{2} \sigma_{s}^{2} J_{A_{R}}
$$

Using the equation (12), the second term $\frac{1}{2} \sigma_{s}^{2} J_{A_{R}}$ in the noise correlation matrix was redefined using a new factor termed as the smoothing factor, computed as in equation (14).

The smoothing factor was modeled based on the fast Fourier transform of the signal received at the receiver and the factor $\mathrm{P}(\mathrm{k})$ as defined in equation (15), which acquired the value as either one or zero. The noise correlation matrix based on the smoothing factor is given as

$$
\psi_{s s}^{A}=\eta \sigma_{s}^{2} J_{A_{R}}+\varpi_{\tau} \sigma_{s}^{2} J_{A_{R}}
$$

where $\varpi_{\tau}$ is the smoothing term and $\eta$ refers to the kernel impulse noise interpolator. The smoothing factor is formulated as

$$
\begin{gathered}
\varpi_{\tau}=[F T(k) * P(k)] \\
P(k)=\left\{\begin{array}{ccc}
1 & ; & \text { if } k<k_{0} \\
0 & ; & \text { if } k>k_{0}
\end{array}\right.
\end{gathered}
$$

where $F T(k)$ refers to the Fast Fourier transform (FFT) of the received signal $k$.The value of $\eta$ varies between 0 and 1 . Substitution of the equation (14) in (13) we got was

$$
\psi_{s S}^{A}=[F T(S) * P(S)] \sigma_{S}^{2} J_{A_{R}}+\eta \sigma_{S}^{2} J_{A_{R}}
$$

Using equation (16), the following computation for the smoothing term dependent noise correlation matrix was made. The output is given as,

$$
\psi_{k k}=K^{C}+\left\lfloor[F T(S) * P(S)] \sigma_{s}^{2} J_{A_{R}}+\eta \sigma_{s}^{2} J\right]
$$

The equalization output was determined using the equation (18) as,

$$
\left.H_{F W-Z F}=\left(K^{C} \psi_{k k}^{-1} C\right)^{-1} K^{c} \mid K K^{C}+\left[[F T(S) * P(S)] \sigma_{s}^{2} J_{A_{R}}+\eta \sigma_{s}^{2} J\right]^{-1}\right]
$$

From the equation (18), it is clear that the equalization output was measured based on the smoothing parameter, variance, identity matrix, and Fast Fourier transform of the received signal. The idea is that the signal from the receiver was initially smoothened through the proposed FWZF equalizer such that the impact of the impulse noise was reduced. Since the channel was a time-varying one, the equalization output was computed as,

$$
H_{F W-Z F}(t+1)=\left(K^{C}(t) \psi_{k k}^{-1}(t) C(t)\right)^{-1} K^{c}\left[\left([F T(S) * P(S)] \sigma_{s}^{2} J_{A_{R}}+\eta \sigma_{s}^{2} J\right)^{-1}\right]
$$

The fusion of the fractional theory is experienced in the equation (19), where the subtraction of the term, $H_{F W-Z F}(t)$ on either side of equation (19) is done. Thus, the equation is,

$$
\begin{aligned}
& H_{F W-Z F}(t+1)-H_{F W-Z F}(t)= \\
& \left(K^{C}(t) \psi_{k k}^{-1}(t) C(t)\right)^{-1} K^{c}\left[\left([F T(S) * P(S)] \sigma_{s}^{2} J_{A_{R}}+\eta \sigma_{s}^{2} J\right)^{-1}\right]-H_{F W-Z F}(t)
\end{aligned}
$$

Let us assume that

$$
\left.\left(K^{C}(t) \psi_{k k}^{-1}(t) C(t)\right)^{-1} K^{c} \mid\left[[F T(S) * P(S)] \sigma_{s}^{2} J_{A_{R}}+\eta \sigma_{s}^{2} J\right)^{-1}\right]-H_{F W-Z F}(t)=Z
$$


and put the assumption in equation (20). Hence, the equation (20) becomes,

$$
H_{F W-Z F}(t+1)-H_{F W-Z F}(t)=Z
$$

It is clearly shown that the equation (21) represents the fractional resemblance; hence, equation (21) is rewritten based on the fractional theory [29] as,

$$
D^{\alpha}\left[H_{F W-Z F}(t+1)\right]=Z
$$

Fractional theory imputes the historical records in the computation found to be free from computation complexity and improves the efficiency of the estimation. In this paper, the historical records of the equalization outputs were retrieved for updating the equalization values in the present, as the major contribution of the research. The equalization output determined diminished the impulse response and enabled higher spectral efficiency. The fractional theory is expanded as follows:

$$
H_{F W-Z F}(t+1)-\alpha H_{F W-Z F}(t)-\frac{1}{2} \alpha H_{F W-Z F}(t-1)-\frac{1}{6} \alpha(1-\alpha) H_{F W-Z F}(t-2)=Z
$$

Rearranging the equation, we got

$$
H_{F W-Z F}(t+1)=\alpha H_{F W-Z F}(t)+\frac{1}{2} \alpha H_{F W-Z F}(t-1)+\frac{1}{6} \alpha(1-\alpha) H_{F W-Z F}(t-2)+Z
$$

Substituting the value of $Z$, we got the following equation as,

$$
\begin{array}{r}
H_{F W-Z F}(t+1)=\alpha H_{F W-Z F}(t)+\frac{1}{2} \alpha H_{F W-Z F}(t-1)+\frac{1}{6} \alpha(1-\alpha) H_{F W-Z F}(t-2)+ \\
\left(K^{C}(t) \psi_{k k}^{-1}(t) C(t)\right)^{-1} K^{c}\left[\left([F T(S) * P(S)] \sigma_{s}^{2} J_{A_{R}}+\eta \sigma_{s}^{2} J\right)^{-1}\right]- \\
H_{F W-Z F}(t)
\end{array}
$$

Equation (25) is the solution of the proposed FWZF strategy of noise mitigation in MIMO-OFDM system.

\section{Results and Discussion}

In this segment, the review of the existing methods through the advantages and disadvantages are enumerated, insisting us a need to establish a new method for noise mitigation in MIMO-OFDM systems and give the overview of the results of the proposed equalizer in MIMO-OFDM systems. The comparative analysis of results demonstrated the effectiveness of FWZF method.

The challenges of the research are summarized with the merits and demerits of the methods. Z. Xie et al. [22] modeled the joint channel estimation and equalization algorithm (JCEE), which was effective for MIMO-SCFDE systems on doubly selective channels. Furthermore, it was suitable to estimate the channel in real time. The disadvantage of the method was the poor average performance of MSE. He, X. et al. [23] developed the MIMO equalizer with fast convergence frequency domain, which had minimal hardware complexity, but the convergence speed was high. Pavan, F.R., et al. [1] modeled a robust blind equalization method that could attain a variant level of steadystate MSE once it returned to ROI, providing a correct first rough equalization before moving to decision-oriented mode. The problem with the method was that it never changed the expected behavior, but avoided the divergence. Pawar, V. et al. [29] developed a doubly selective channel equalizer called as the blind time domain equalizer to reduce the time average of the correlation matrix, which led to the formation of the computationally efficient algorithm. However, the increase in multipath has never affected Bit Error Rate (BER) performance. Samara, L. et al. [25] developed a joint phase noise mitigation and channel equalization technique, using significant reduction in the entries in the equalizer matrix with minimal loss of performance. However, at minimal SNR, there was domination in the channel effect due to noise. P. Zhe et al. [24] used a Robust Single-Carrier Frequency Domain Equalization, which reduced the complexity, but with the increase of the TS there was a performance gap causing the estimation of the power channel. Liu, W., [26] developed an adaptive algorithm evaluated in quaternions, which proved to have better convergence. However, the initialization of the beam forming algorithm and equalization at the same time depends on the MIMO structure.Conventional techniques developed for Gaussian noise do not work well under impulse noise.

\subsection{Experimental setup}

The execution of the methods was achieved using MATLAB and the learning of the techniques was accomplished by the performance metrics, such as SER and BER and the performance was analyzed the under fading and impulse noise environment.

\subsection{Performance metrics}

In this section, two performance metrics BER\& SER have been chosen to evaluate the performance of various methods as they are widely represented metrics in literature.

a. BER: It is the ratio of the bit errors to the total bits received, transmitted, or processed in a particular time period. It is formulated as

$$
B E R=\frac{1}{2} \operatorname{erfc} \sqrt{\frac{\varepsilon}{\mathrm{N}}}
$$

b. SER: It is the measure of the interference and formulated as the expectation over the symbols as given by,

$$
S E R=E E\left[q q\left(\frac{W W+D I}{\sqrt{\sigma_{\text {NOISE }}}}\right)\right]
$$

where, $E E()$ symbolizes the expectation, qq is the Q function, $\sigma_{\text {NOISE }}$ is the variance of the noise, $D I$ is the dominant interference, and $W W$ is the weight.

Table 1. Simulation Parameters

\begin{tabular}{lc}
\hline \multicolumn{1}{c}{ Parameters } & Value \\
\hline FFT size & 256 \\
Number of subcarrier & 256 \\
Cyclic prefix size & 144 \\
Modulation type & QPSK \\
OFDM symbol duration & $1.13 \mathrm{~ms}$ \\
Number of OFDM frames & 256 \\
Number of transmit and receive antennas & 4 \\
\hline
\end{tabular}

\subsection{Comparative methods}

Methods used for comparison included: Joint channel estimation and equalization (JCEE) [23], Adjustable MIMO decision feedback equalization (DFE)[15], Zero Forcing Smoothing term based Minimum Mean Output Energy (ZF - 


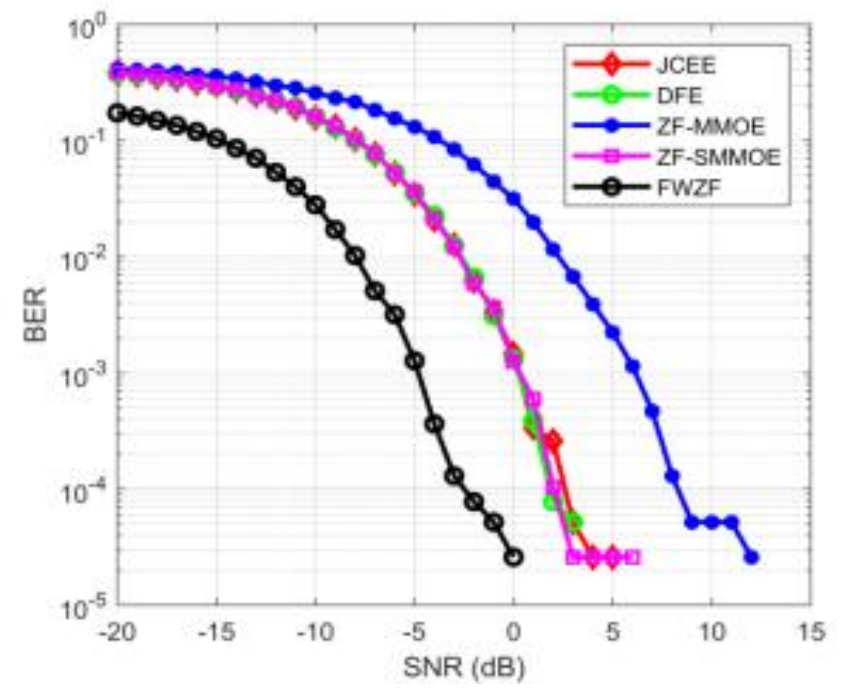

(a)

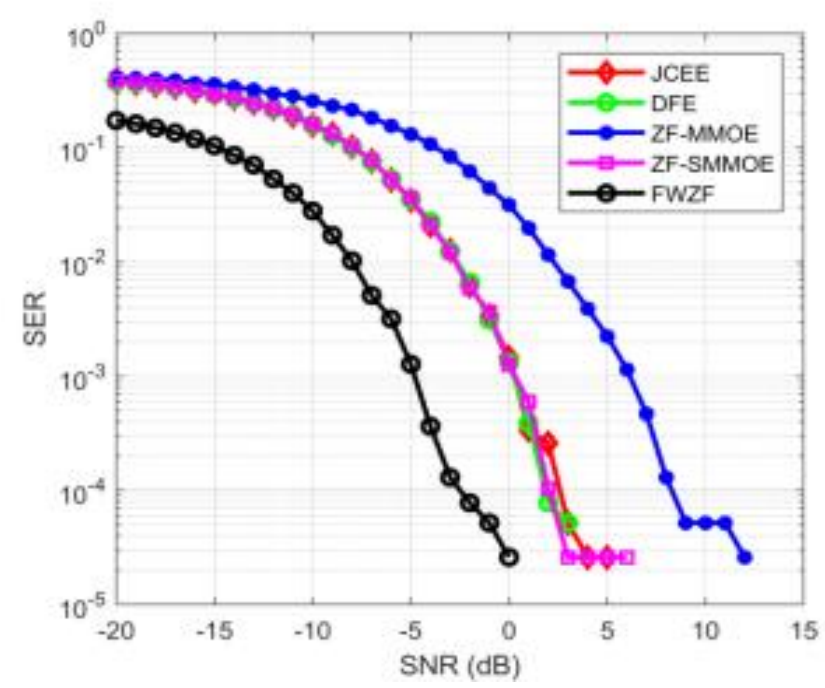

(b)

Fig. 2. Comparative analysis using antenna=4 in Rayleigh channel, (a) BER, (b) SER

SMMOE) [2] and Zero Forcing Minimum Mean Output Energy (ZF-MMOE) [11].

\subsection{Performance analysis}

In this paper, the performance analysis of the methods was deliberated using four, five, and six antennas in the presence of Rayleigh and Rician Channels.

\subsubsection{Analysis using four antennas and channel as Rayleigh}

Figure 2 shows the performance analysis of the methods in the presence of four antennas and the channel considered was Rayleigh. figure 2 (a) shows the performance analysis based on BER and figure 2 (b) shows the analysis as a function of SER so that the analysis progressed with respect to the signal to noise ratio (SNR). The SNR interval was set between -20 and $15 \mathrm{~dB}$ and analysis was performed. When SNR was $-20 \mathrm{~dB}$, the BER of the methods, JCEE, DFE, ZF-MMOE, ZF-SMMOE and FWZF was $0.3841,0.3808,0.4176,0.3835$ and 0.1733 respectively. It was observed that the BER of the methods decreased with increasing SNR value. However, the proposed FWZF technique acquired the minimum BER value compared to existing methods. Similarly, the SER based on the SNR in figure 2 (b) was discussed below: When the SNR was $-20 \mathrm{~dB}$, the SER of the methods, JCEE, DFE, ZF-MMOE, ZF-SMMOE and FWZF was $0.3841,0,3808,0.4176,0.3835$ and 0.1733 , respectively. It was observed that the SER of the methods decreased with increasing SNR value. However, the proposed FWZF technique acquired the minimal SER value compared to existing methods.

\subsubsection{Analysis using four antennas and channel as Rician}

Figure 3 shows the performance analysis of the methods in the presence of four antennas and the channel considered is Rician. Figure 3 (a) shows the performance analysis based on BER and figure 3 (b) shows the analysis that depends on SER so that the analysis proceeded with respect to the signal-tonoise ratio (SNR). The SNR interval was set between -20 and $15 \mathrm{~dB}$ and analysis was performed. When SNR was $-20 \mathrm{~dB}$, the

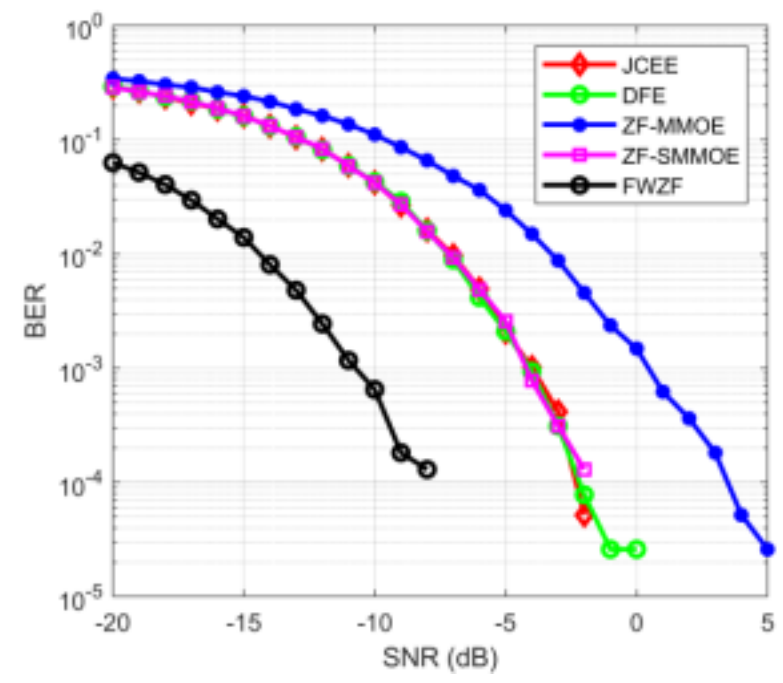

(a)

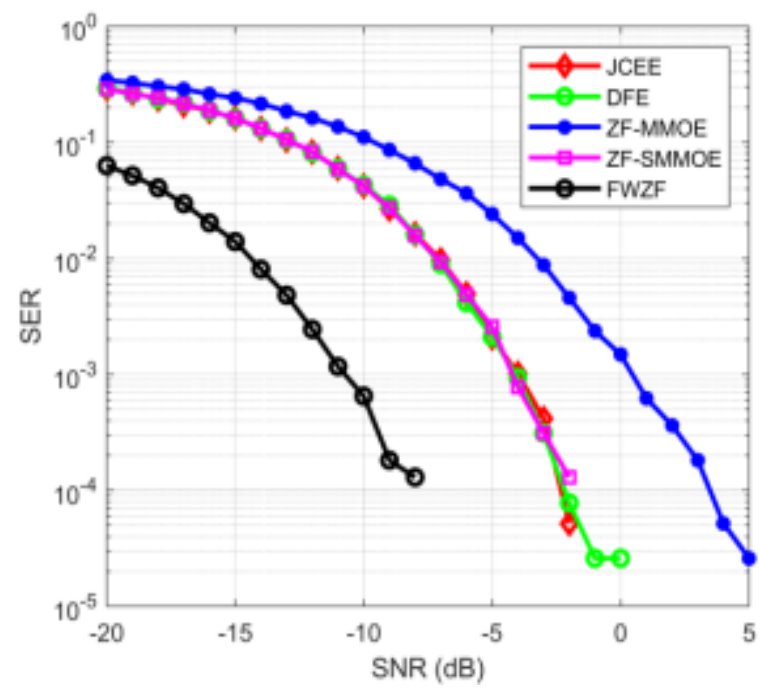

(b)

Fig. 3. Comparative analysis using antenna=4 in Rician channel, (a) BER, (b) SER 


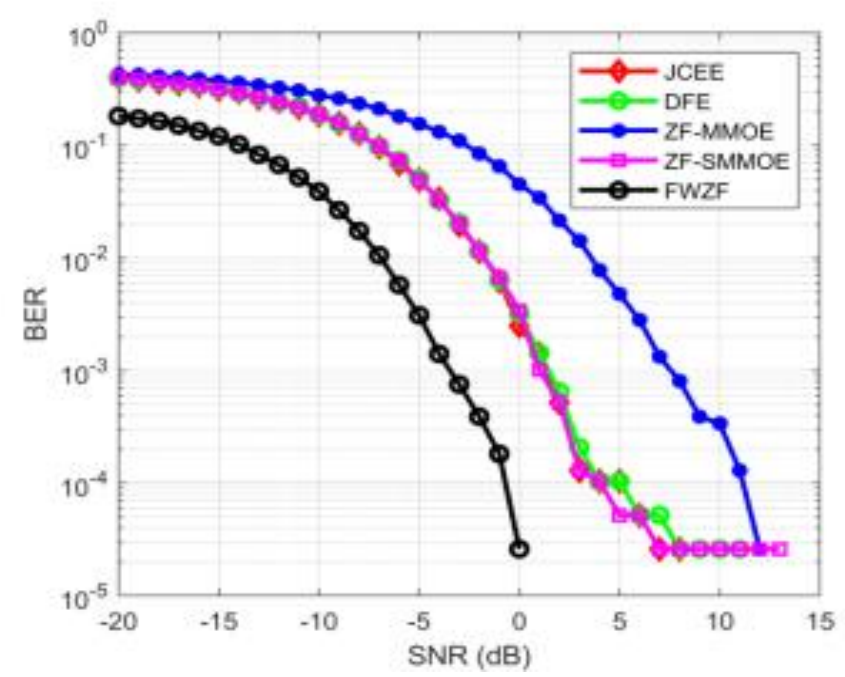

(a)

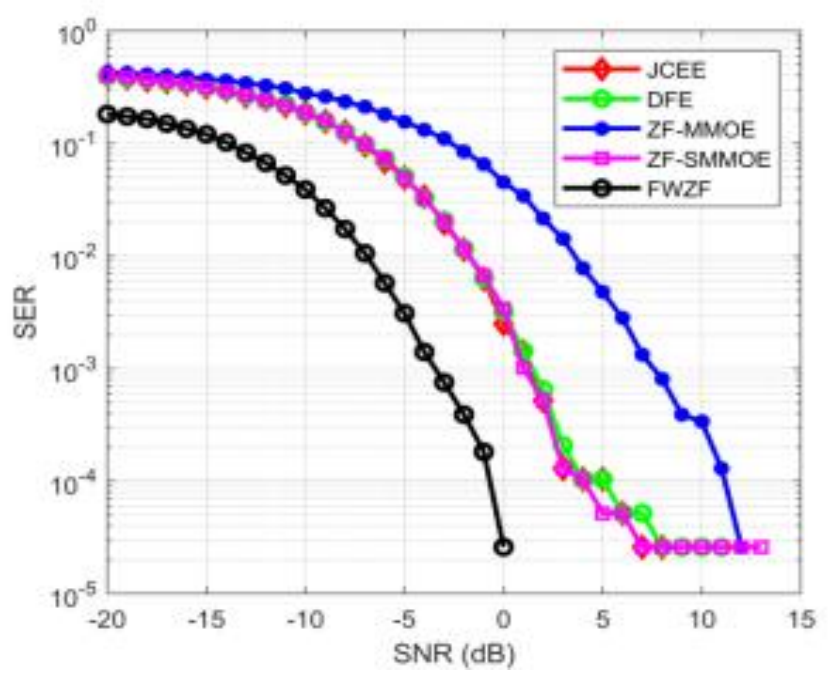

(b)

Fig. 4. Comparative analysis using antenna=5 in Rayleigh channel, (a) BER, (b) SER

BER of the methods, JCEE, DFE, ZF-MMOE, ZF-SMMOE and FWZF was $0.2897,0.2929,0.3440$, respectively. 0.2883 and 0.0630 , respectively. It was found observed that the BER of the methods decreased with the increasing SNR value. However, the proposed FWZF technique acquired the minimum BER value compared to existing methods. Similarly, the SER based SNR in figure 3 (b) is discussed below: When the SNR was $-20 \mathrm{~dB}$, the SER of the methods, JCEE, DFE, ZFMMOE, ZF-SMMOE and FWZF was 0.2897, 0.2929, 0.3440, 0.2883 and 0.0630 , respectively. It was observed that the SER of the methods decreased with increasing SNR value. However, the proposed FWZF technique acquired the minimum SER value compared to existing methods.

\subsubsection{Analysis using five antennas and channel as Rayleigh}

Figure 4 shows the analysis of the performance of the methods in the presence of five antennas and the channel

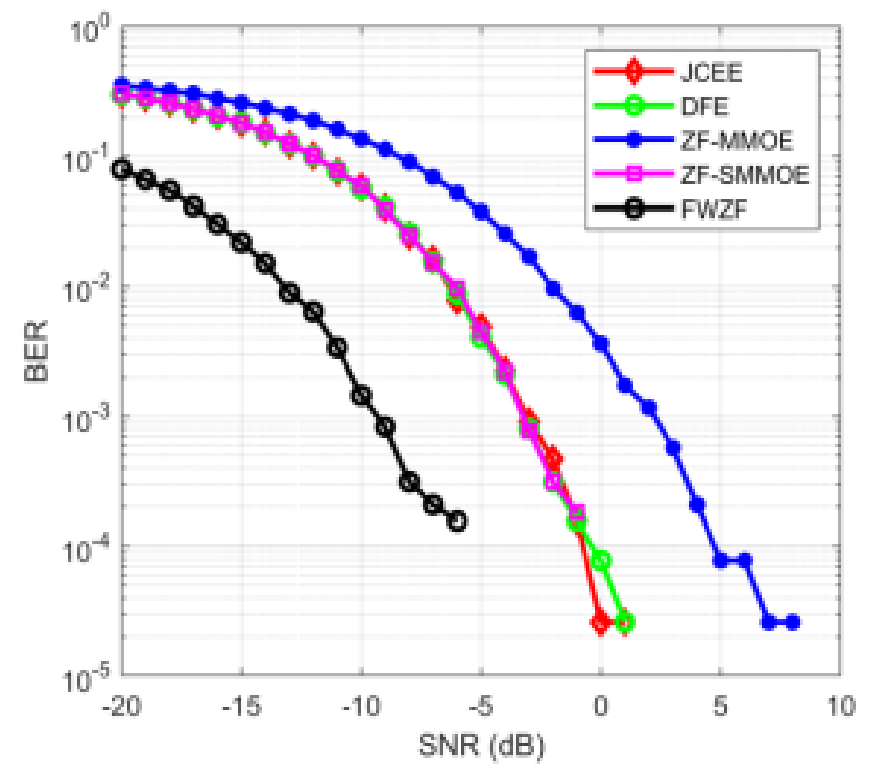

(a) considered was Rayleigh. Figure 4 (a) shows the performance analysis based on BER and figure 4 (b) shows the analysis as a function of SER so that the analysis proceeded with respect to the signal-to-noise ratio (SNR). The SNR interval was set between -20 and $15 \mathrm{~dB}$ and analysis was performed. When SNR was $-20 \mathrm{~dB}$, the BER of the methods, JCEE, DFE, ZFMMOE, ZF-SMMOE and FWZF was 0.4008, 0.3965, 0.4251, 0.3985 and 0.1817 , respectively. It was observed that the BER of the methods decreased with the increasing SNR value. However, the proposed FWZF technique acquired the minimum BER value compared to existing methods. Similarly, the SER based SNR in figure 4 (b) is discussed below: When the SNR was -20dB, the SER of the methods, JCEE, DFE, ZFMMOE, ZF-SMMOE and FWZF was 0.4008, 0.3965, 0.4251, 0.3985 and 0.1817 , respectively. It was observed that the SER of the methods decreased with the increasing SNR value. However, the proposed FWZF technique acquired the minimum SER value compared to existing methods.

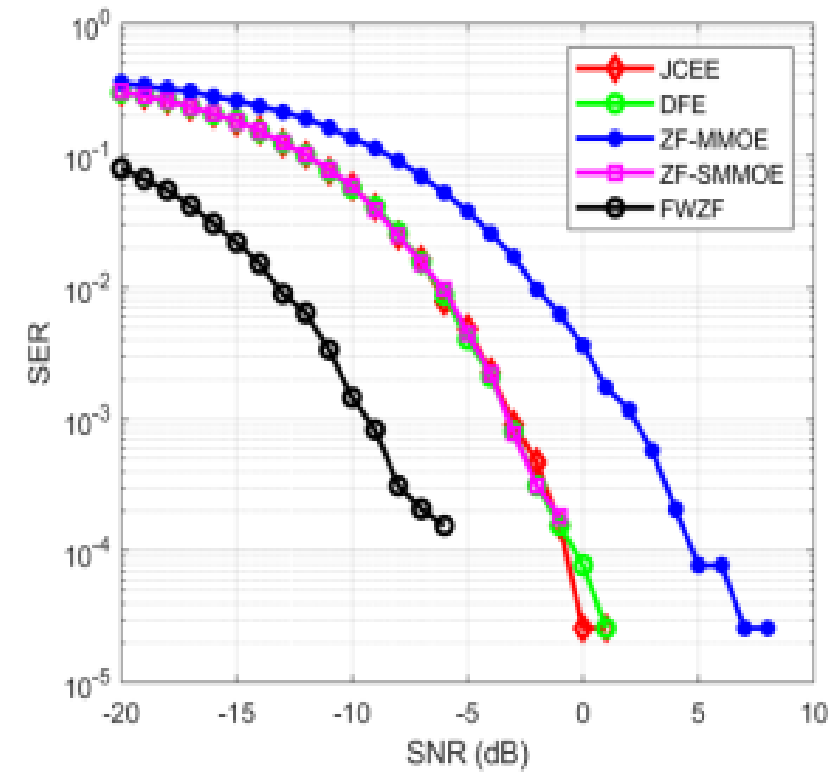

(b)

Fig. 5.Comparative analysis using antenna=5 in Rician channel, (a) BER, (b) SER 


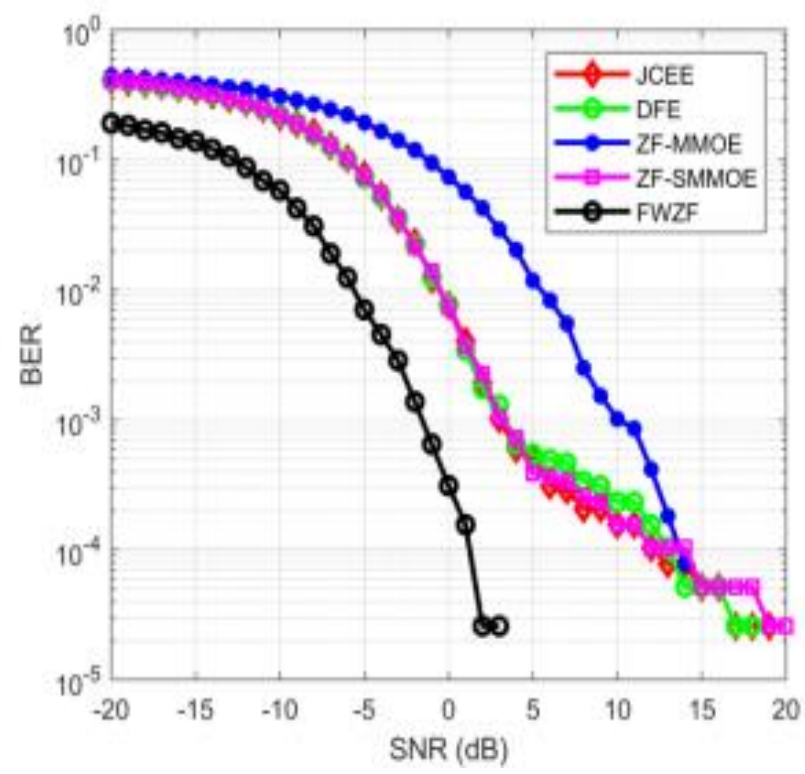

(a)

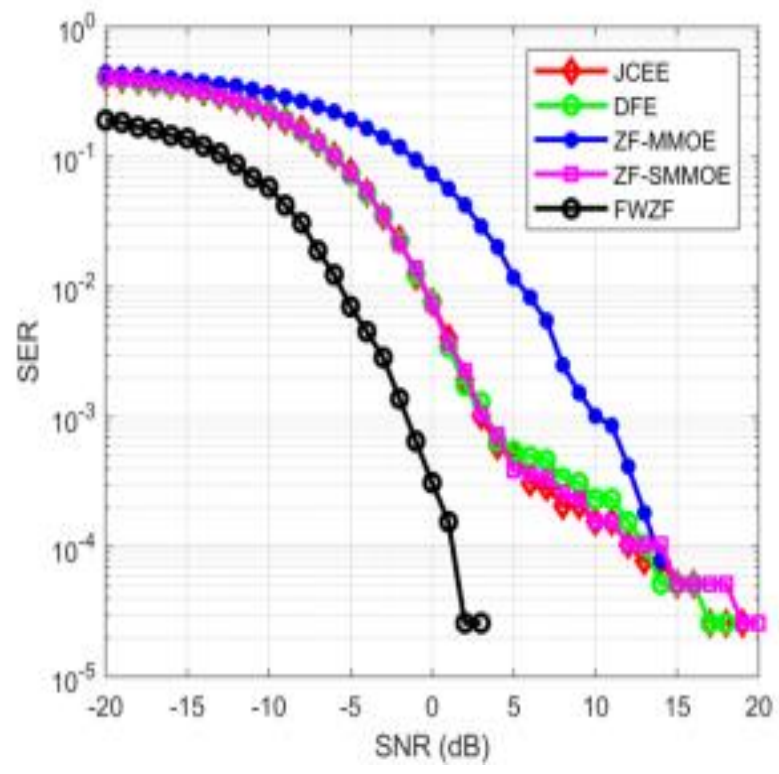

(b)

Fig.6. Comparative analysis using antenna=6 in Rayleigh channel, (a) BER, (b) SER

\subsubsection{Analysis using five antennas and channel as Rician}

Figure 5 shows the performance analysis of the methods in the presence of five antennas and the channel considered is Rician. Figure 5 (a) shows the performance analysis based on BER and figure 5 (b) shows the analysis that depends on SER so that the analysis progresses with respect to the signal / noise ratio (SNR). The SNR interval is set between -20 and $15 \mathrm{~dB}$ and analysis is performed. When SNR is $-20 \mathrm{~dB}$, the BER of the methods, JCEE, DFE, ZF-MMOE, ZF-SMMOE and FWZF is $0.30260 .3015,0.3561,0.3072$ and 0.0796 respectively. It is observed that the BER of the methods decreases with increasing SNR value. However, the proposed FWZF technique acquired the minimum BER value compared to existing methods. Similarly, the SER based on SNR in figure 5 (b) is analyzed below: when the SNR is $-20 \mathrm{~dB}$, the SER of the

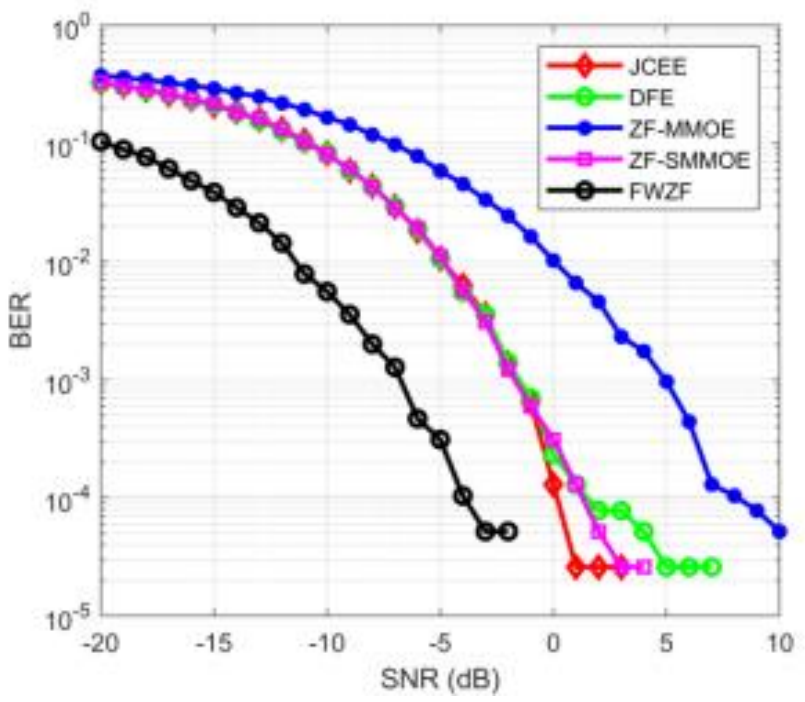

(a) methods, JCEE, DFE, ZF-MMOE, ZF-SMMOE and FWZF is $0.3026,0,3015,0.3561,0.3072$ and 0.0796 , respectively. It is observed that the SER of the methods decreases with increasing SNR value. However, the proposed FWZF technique has acquired the minimum SER value compared to existing methods.

\subsubsection{Analysis using six antennas and channel as Rayleigh}

Figure 6 shows the analysis of the performance of the methods in the presence of six antennas and the channel considered was Rayleigh. Figure 6 (a) shows the performance analysis based on BER and figure 6 (b) shows the analysis dependent on SER so that the analysis progressed with respect to the signal-to-noise ratio (SNR). The SNR interval was set between -20 and $15 \mathrm{~dB}$ and analysis was performed. When

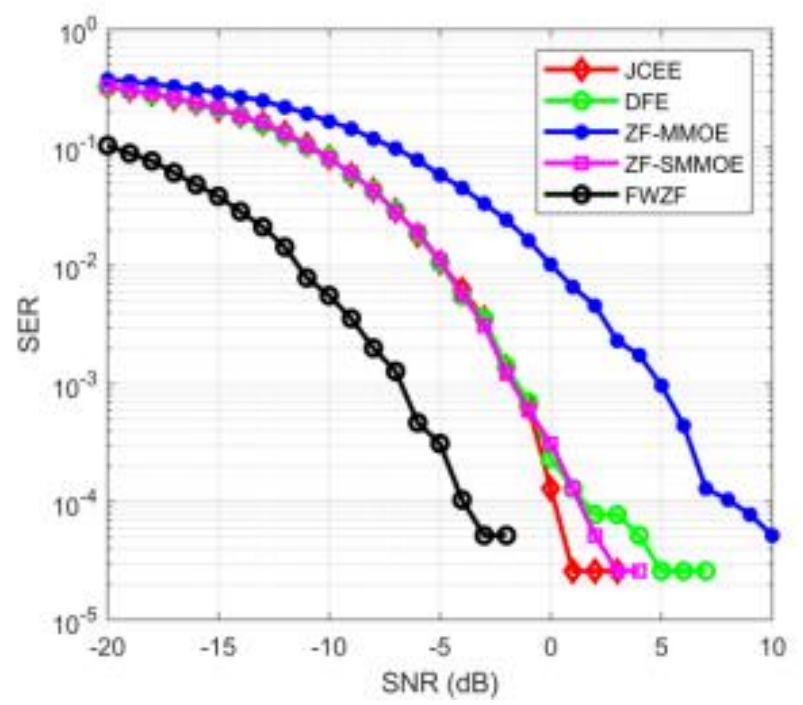

(b)

Fig. 7. Comparative analysis using antenna $=6$ in Rician channel, (a) BER, (b) SER 
SNR was $-20 \mathrm{~dB}$, the BER of the methods, JCEE, DFE, ZFMMOE, ZF-SMMOE and FWZF was 0.4106, 0.4099, 0.4403, 0.4068 and 0.1913 , respectively. It was observed that the BER of the methods decreased with the increasing SNR value. However, the proposed FWZF technique acquired the minimum BER value compared to existing methods. Similarly, the SER based on SNR in figure 6 (b) is discussed below: when the SNR was -20dB, the SER of the methods, JCEE, DFE, ZFMMOE, ZF-SMMOE and FWZF was 0.4106, 0.4099, 0.4403, 0.4068 and 0.1913 , respectively. It was observed that the SER of the methods decreased with the increasing SNR value. However, the proposed FWZF technique acquired the minimum SER value compared to existing methods.

\subsubsection{Analysis using six antennas and channel as Rician}

Figure 7 shows the performance analysis of the methods in the presence of six antennas and the channel considered was Rician. Figure 7 (a) shows the performance analysis based on BER and figure 7 (b) shows the analysis that depended on SER so that the analysis progressed with respect to the signal-tonoise ratio (SNR). The SNR interval was set between -20 and $15 \mathrm{~dB}$ and analysis was performed. When SNR was $-20 \mathrm{~dB}$, the BER of the methods, JCEE, DFE, ZF-MMOE, ZF-SMMOE and FWZF was $0.3278,0.3251,0.3749,0.3296$ and 0.1038 respectively. It was observed that the BER of the methods decreased with the increasing SNR value. However, the proposed FWZF technique acquired the minimum BER value compared to existing methods. Similarly, the SER based on SNR in figure 7 (b) is discussed below: when the SNR was $20 \mathrm{~dB}$, the SER of the methods, JCEE, DFE, ZF-MMOE, ZFSMMOE and FWZF was 0.3278, 0.3251, 0.3749, 0.3296 and 0.1038 , respectively. It was observed that the SER of the methods decreased with the increasing SNR value. However, the proposed FWZF technique has acquired the minimum SER value compared to existing methods.

\subsection{Comparative Discussion}

Table 2 presents the comparative analysis of the methods in the Rayleigh channel. The BER of the methods, JCEE, DFE, ZF-MMOE, ZF-SMMOE and FWZF was 0.2897, 0.2929, $0.3440,0.2883$ and 0.0630 respectively. The SERs of the methods, JCEE, DFE, ZF-MMOE, ZF-SMMOE and FWZF were $0.3278,0.3251,0.3749,0.3296$ and 0.1038 respectively. It is clear that the proposed FWZF method outperformed the existing methods with the minimum BER and SER values, respectively.

Table 2. Comparative discussion using the ZF methods in Rayleigh Channel

\begin{tabular}{lccccc}
\hline Methods & JCEE & DFE & ZF-MMOE & ZF-SMMOE & FWZF \\
\hline BER & 0.2897 & 0.2929 & 0.3440 & 0.2883 & 0.0630 \\
& & & & & \\
SER & 0.3278 & 0.3251 & 0.3749 & 0.3296 & 0.1038
\end{tabular}

Table 3 presents the comparative analysis of the methods in the Rician channel. The BER of the methods, JCEE, DFE, ZF-MMOE, ZF-SMMOE and FWZF was 0.3026, 0.3015, $0.3561,0.3072$ and 0.0796 respectively. The SERs of the methods, JCEE, DFE, ZF-MMOE, ZF-SMMOE and FWZF were $0.3841,0.3808,0.4176,0.3835$ and 0.1733 respectively. It is clear that the proposed FWZF method outperformed the existing methods with the minimum BER and SER values, respectively.

Table 3. Comparative discussion using the ZF methods in Rician Channel

\begin{tabular}{lccccc}
\hline Methods & JCEE & DFE & ZF-MMOE & ZF-SMMOE & FWZF \\
\hline BER & 0.3026 & 0.3015 & 0.3561 & 0.3072 & 0.0796 \\
& & & & & \\
SER & 0.3841 & 0.3808 & 0.4176 & 0.3835 & 0.1733 \\
\hline
\end{tabular}

\section{Conclusion}

The impulse noise in the MIMO-OFDM systems degrades the performance of the system affecting the spectral efficiency in the network. Thus, the mitigation of the impulse noise is essential. In this paper an impulse noise mitigation strategy based on the fractional weighted zero-forcing (FWZF) equalizer is presented and the equalizer outperformed the existing linear equalizer. The proposed Fractional Weighted Zero-forcing equalizer is the combination of the fractional concept in the Zero-Forcing equalizer. The successive step in the formulation of the equalizer demolishes the impulse noise at the receiver terminal and enhances the communication performance thereby, increasing the spectral efficiency without any computational complexity. The performance of the noise mitigation in the MIMO-OFDM systems was analyzed using the comparative measures, such as BER and SER and the analysis was progressed both in the Rayleigh and Rician channel. The minimal value of BER and SER was reported for the proposed FWZF technique in the Rayleigh channel, about 0.063 , and 0.1038 , respectively. The proposed equalizer outperformed the existing methods.

\section{References}

1. R. Pavan, M.T. Silva, M.D. Miranda, A numerically robust blind equalization scheme applied to MIMO communication systems, J. Franklin Inst. 355 (2018) 596-624.

2. S.P. Girija and K.D. Rao, Smoothing term based noise correlation matrix construction for MIMO-OFDM wireless networks for impulse noise mitigation, TENCON-2015, IEEE Region 10 Conference, Macao, 2015

3. Q. Liu, L. Yang, K. Li, Decorrelating bootstrap equalizer for timevariation suppression of MIMO channel, Signal Process. 86 (2006) 1509-1517.

4. G. Faria, J.A. Henriksson, E. Stare, P. Talmola, DVB-H: Digital broadcast services to handheld devices, Proceed. IEEE, 94 (2006) 194-209.

5. R. Arablouei, and K. Doğançay, Low-complexity adaptive decision- feedback equalization of MIMO channels, Signal Process. 92 (2012) 1515-1524.

6. N. Rožić, P. Banelli, D. Begušić, J. Radić, Multiple-threshold estimators for impulsive noise suppression in multicarrier communications, IEEE Trans. Signal Process. 66 (2018) 1619. 1633.

7. Y. Chen, Y. Zhang, H. Shu, J. Yang, L. Luo, J. Coatrieux, Q. Feng, Structure-Adaptive Fuzzy Estimation for Random-Valued Impulse Noise Suppression, IEEE Transact. Circ. Syst. Video Technol. 28 
(2018) 414 - 427.

8. H. Liu, Y. Li, Y. Zhou, H. Chang, T. Truong, Impulsive noise suppression in the case of frequency estimation by exploring signal sparsity, Digital Signal Process. 57 (2016) 34-45.

9. H. Hosseini, F. Hessar, F. Marvasti, Real-time impulse noise suppression from images using an efficient weighted-average filtering, IEEE Signal Process. Lett. 22 (2015) 1050-1054.

10. A. Bansal and A.K. Kohli, Suppression of impulsive noise in OFDM system using imperfect channel state information, Optik 127 (2016) 2111-2115.

11. D. Darsena, G. Gelli, F. Melito, F. Verde, A. Vitiello, Impulse noise mitigation for MIMO-OFDM wireless networks with linear equalization, IEEE Int. Workshop Meas. Net. (M\&N), Naples, 2013, pp. 94-99.

12. N. Benvenuto and S. Tomasin, On the comparison between OFDM and single carrier modulation with a DFE using a frequency-domain feed-forward filter, IEEE Trans. Commun. 50 (2002) 947-955.

13. Z. Xie, X. Chen, X. Liu, A virtual pilot-assisted channel estimation algorithm for MIMO-SCFDE systems over fast time-varying multipath channels, IEEE Trans. Veh. Technol. 67 (2018) 49014909 .

14. Y. Zhang, Y.V. Zakharov, J. Li, Soft-decision-driven sparse channel estimation and turbo equalization for MIMO underwater acoustic communications, IEEE Access 6 (2018) 4955-4973.

15. N. Al-Dhahir, A.F. Naguib, A.R. Calderbank, Finite-length MIMO decision feedback equalization for space-time blockcoded signals over multipath-fading channels," IEEE Trans. Vehi. Technol. 50 (2001) 1176-1182.

16. M. Sellathurai and S. Haykin, A simplified diagonal BLAST architecture with iterative parallel interference cancellation receivers, IEEE Int. Conf. Commun. 10 (2001) 3067-3071.

17. H. Luo, R.-W. Liu, X. Lin, X. Li, The autocorrelation matching method for distributed MIMO communications over unknown FIR channels, IEEE Int. Conf. Acoustics Speech Signal Process. 4 (2001) 2161-2164.

18. A. Maleki-Tehrani, B. Hassibi, J.M. Cioffi, Adaptive equalization of multiple-input multiple-output (MIMO) channels, IEEE Int. Conf. Commun. 3 (2000) 1670-1674.

19. A. Aminjavaheri, A. Farhang, B. Farhang-Boroujeny, Filter bank multicarrier in massive MIMO: Analysis and channel equalization, IEEE Trans. Signal Process. 66 (2018) 3987 - 4000.

20. J. Coon, M. Sandell, M. Beach, J. McGeehan, Channel and noise variance estimation and tracking algorithms for unique-word based single carrier systems, IEEE Trans. Wireless Commun. 5 (2006) 1488-1496.

21. N. Benvenuto, R. Dinis, D.D. Falconer, S. Tomasin, Single carrier modulation with nonlinear frequency domain equalization: An ided whose time has come again, Proc. IEEE 98 (2010) 69-96.

22. Z. Xie, X. Chen, X. Liu, Joint channel estimation and equalization for MIMO-SCFDE systems over doubly selective channels, J. Commun. Net. 19 (2017) 627-636.

23. X. He, Y. Weng, J. Wang, Z. Pan, Fast convergent frequencydomain MIMO equalizer for few-mode fiber communication systems, Optics Commun. 409 (2018) 131-136.

24. P. Zhe, Y. Zhu, K.B. Letaief, Robust single-carrier frequency domain equalization for broadband MIMO systems with imperfect channel estimation, IEEE Trans. Wireless Commun. 17 (2018) 4432-4446.

25. L. Samara, A.O. Al-Abbasi, R. Hamila, N. Al-Dhahir, Reducedcomplexity sparsity-aware joint phase noise mitigation and channel equalization in OFDM receivers, Phys. Commun. 30 (2018) 50-57.

26. W. Liu, Channel equalization and beamforming for quaternionvalued wireless communication systems, J. Franklin Inst. 354 (2017) 8721-8733.

27. K. Ramadan, M.I. Dessouky, S. Elagooz, M. Elkordy, F.A. ElSamie, Equalization and carrier frequency offset compensation for underwater acoustic OFDM systems, Annals Data Sci. 5 (2018) 259-272.

28. P.R. Bhaladhare, A clustering approach for the l-diversity model in privacy preserving data mining using fractional calculus-bacterial foraging optimization algorithm, Adv. Comput. Eng. 2014 (2014) 396529.

29. V. Pawar, R. Pawar, K. Nai, Blind time-domain equalizer for doubly-selective channel with reduced time averaging and computational complexity, AEU-Int. J. Electronics Commun. 94 (2018) 187-198. 